\title{
Resonantly Enhanced Silicon Photonics Mach-Zehnder Modulator
}

\author{
Kasper Van Gasse, Gunther Roelkens \\ Photonics Research Group, \\ Department of Information Technology, \\ Ghent University - imec, Ghent, Belgium \\ Email: kasper.vangasse@ugent.be
}

\author{
Qiangsheng Huang \\ Centre for Optical and Electromagnetic Research, \\ State Key Laboratory \\ for Modern Optical Instrumentation, \\ Zhejiang University, Hangzhou 310058, China
}

\begin{abstract}
In this work we present the design, fabrication and characterization of a silicon photonics Mach-Zehnder modulator (MZM) with resonant electrode. The device shows an enhanced modulation response in the $20 \mathrm{GHz}$ frequency range compared to a standard traveling-wave MZM.
\end{abstract}

\section{INTRODUCTION}

Over the last years a great amount of research has been dedicated to the development of high-bandwidth optical modulators on integrated photonic platforms, such as silicon photonics, InP photonics and hybrid InP/Si photonics. One type of modulator that has been successfully developed during the past few years is the depletion-type Si Mach-Zehnder modulator (MZM) [1] ${ }^{-}[2]$. These have been designed with (broadband) baseband applications in mind. Recently integrated microwave photonics has gained a lot of interest [3]. Integrated microwave photonics is relevant for wireless applications in next generation mobile networks(5G). Such applications often require only a narrow bandwidth however around a high carrier frequency, typically in the $20-60 \mathrm{GHz}$ frequency range. One of the main challenges in the design of a Si depletion type MZM is the traveling wave electrode. This electrode design is usually focused on having a flat response over a wide frequency range, for baseband applications. An interesting alternative for microwave photonic applications is to use a resonant electrode structure, leading to a resonantly Enhanced MZM (RE-MZM). This approach has been explored on the lithiumniobate platform and has shown great promise[4]. However, the modulation efficiency of a lithium-niobate modulator is about $10 \mathrm{Vcm}$. Electrodes which are resonant at frequencies higher than $20 \mathrm{GHz}$ have lengths in the order of millimeters, and it is therefore interesting to use resonant electrodes on a platform with a smaller VpiLpi such as Si photonics. In this paper the design, fabrication and characterization of a resonantly-enhanced MZM is discussed.

\section{RE-MZM DESIGN}

The Si MZM with resonant electrode was realized on the imec iSiPP25G platform. This silicon photonics platform offers a Si depletion-type phase shifter and travelling wave MZM (TW-MZM) as standard component. The optical phase shifter is realized by lateral pn-doping of a rib waveguide. The $\mathrm{Si}$ waveguide is $450 \mathrm{~nm}$ wide and $220 \mathrm{~nm}$ thick with a 2

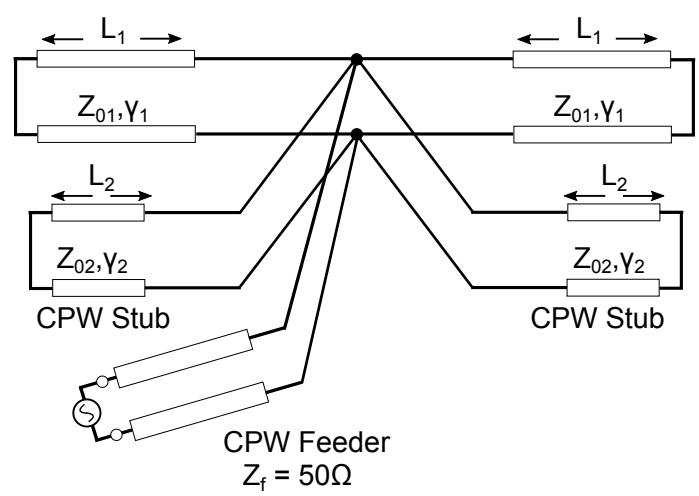

Fig. 1. The equivalent circuit used to design the resonant electrode.

$\mu \mathrm{m}$ thick BOX layer underneath. On top of the waveguide a $1.5 \mu \mathrm{m}$ dielectric layer stack is deposited. A $0.5 \mu \mathrm{m}$ thick $\mathrm{Cu}$ layer, $1 \mu \mathrm{m}$ above the $\mathrm{Si}$ waveguide, is used to form the coplanar waveguide electrode. The doped waveguide regions are connected to the metal layer with tungsten vias. The doped waveguide is placed in between the signal and one of the ground lines of the CPW. The MZI uses a $1 \mathrm{X} 2$ and a $2 \mathrm{X} 2$ MMI at the input and output respectively. Only the top arm contains the resonant electrode phase shifter, the bottom arm contains a static p-n phase shifter which can be used to set the bias point of the modulator and which balances the losses in both arms of the MZM. The optical pathlength in both arms of the MZM is identical, therefore the optical bandwidth of the modulator is only limited by the grating couplers. For the design of the RE-MZM a custom co-planar waveguide with optical phase shifter was designed. The goal of the electrode is to have enhanced modulation performance in the 25-30 $\mathrm{GHz}$ band, which is a frequency range of great interest for $5 \mathrm{G}$ wireless networks. A design with shorted termination and stubs for impedance matching was chosen. First the mode of the electrical wave was simulated with EM simulation software. This is then used to determine the impedance and propagation constant of an equivalent transmission line circuit. For the stubs and the feedline a transmission line was designed with low loss and a characteristic impedance of $50 \mathrm{Ohm}$. The transmission lines were further optimized for minimal loss 


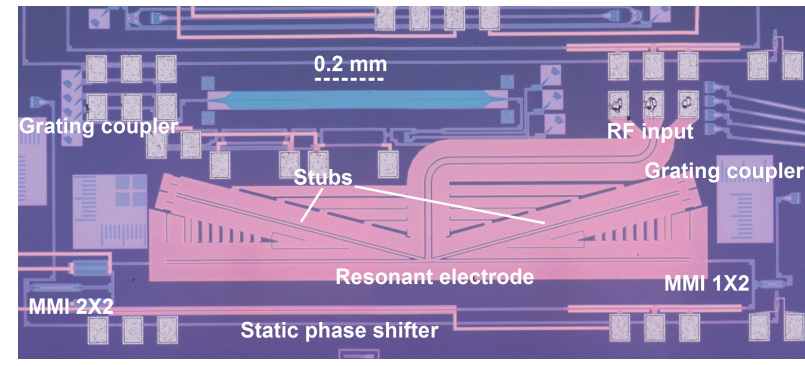

Fig. 2. Microscope figure of the MZM with resonant electrode.

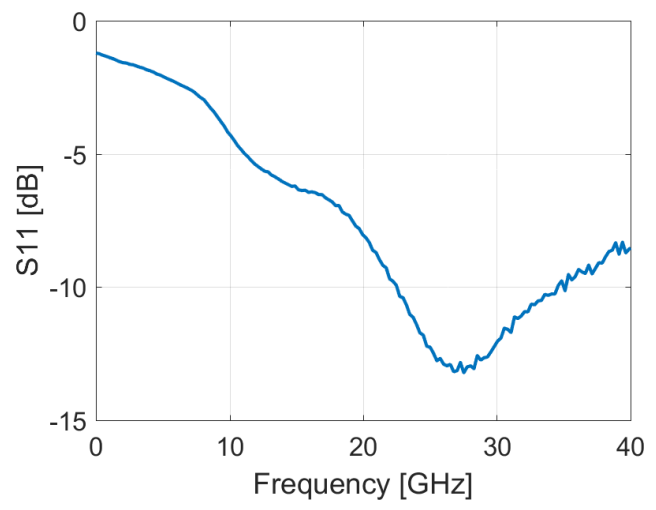

Fig. 3. Measured S11 of the RE-MZM.

and constant impedance at higher frequencies. The impedance at the feedpoint of the structure can be calculated using the simulated transmission line parameters and transmission line model given by the formula:

$$
Z_{\text {in }}=\frac{\mathrm{Z}_{1} \tanh \left(\gamma_{1} L_{1}\right) \mathrm{Z}_{2} \tanh \left(\gamma_{2} L_{2}\right)}{\mathrm{Z}_{1} \tanh \left(\gamma_{1} L_{1}\right)+\mathrm{Z}_{2} \tanh \left(\gamma_{2} L_{2}\right)}
$$

The length of the stubs was chosen such that the feedpoint impedance is $50 \mathrm{Ohm}$ at resonance. The length of the modulating electrode was design to have a strong electro-optic response (i.e. a resonance) at $25-30 \mathrm{GHz}$. The resonant phase shifter is $1.6 \mathrm{~mm}$ long and has an optical loss of $4.8 \mathrm{~dB}$. The $V_{p i} L_{p i}$ of the phase shifter is $1 V \mathrm{~cm}$. To determine the resulting optical modulation efficiency we need to account for velocity matching and the voltage distribution along the length of the electrode. This can be analytically caclulated using the transmission line model as was done in [4]. A microscope picture of the realized device is shown in Fig. 2.

\section{Measurements}

To characterize the modulator an electric and electro-optical measurement was performed. The S11-parameter of the modulator was measured using a Keysight PNA-X and the result is shown in Fig. 3. We see a clear dip in the reflection spectrum between 20 and $30 \mathrm{GHz}$. Because the electrodes are terminated with a short the reflection approaches $0 \mathrm{~dB}$ at low frequencies. We then measured the S21 of the modulator also using a Keysight PNA-X, a Santec tunable laser and $\mathrm{u}^{2} \mathrm{t}$ $40 \mathrm{GHz}$ photodetector. The output of the laser was coupled

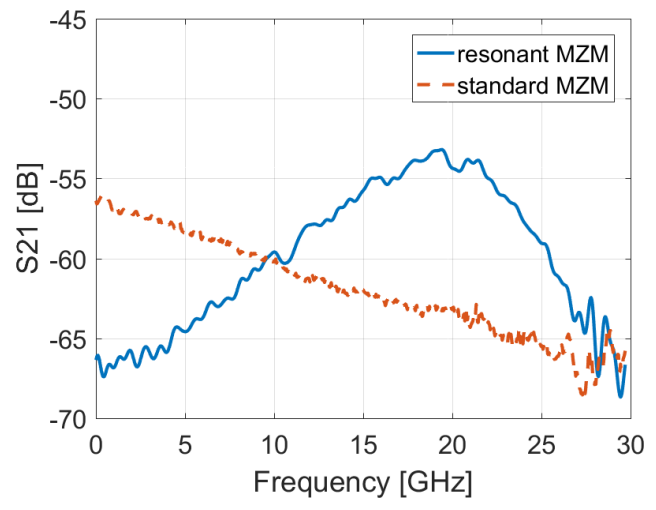

Fig. 4. Measured S21 of the RE-MZM and TW-MZM with termination.

into the PIC using a cleaved SMF through an on-chip vertical grating coupler. The MZI was biased at the quadrature point and driven with a $-5 \mathrm{dBm}$ RF signal. The light is coupled out using standard single mode fiber. The output was amplified with an EDFA to compensate for the coupling losses. The resulting S21 response is shown in Fig. 4. During the same measurement a TW-MZM on the same die (with a phase shifter length of $1.34 \mathrm{~mm}$ and a $30 \mathrm{Ohm}$ termination ) was measured as reference and is also shown in Fig. 4. The TW-MZM was designed to have an maximum bandwidth with flat response. The electro-optic response of the RE-MZM shows a broad resonance around $20 \mathrm{GHz}$. This shift from the design can be explained by a microwave refractive index deviating from the simulation, due to dimensional variations in the modulator cross-section. The RE-MZM outperforms the standard MZM by more than $6 \mathrm{~dB}$ at $20 \mathrm{GHz}$. The $3 \mathrm{~dB}$ width of the resonance is approx $8 \mathrm{GHz}$. The RE-MZM outperforms the TW-MZM in the entire interval.

\section{CONCLUSION}

In this work we have presented the design of a RE-MZM and presented measurement results of the fabricated device. The electro-optic response shows an enhanced modulation in a $8 \mathrm{GHz}$ band around $20 \mathrm{GHz}$ and outperforms a TW-MZM in that frequency range. To the best of our knowledge this is the first RE-MZM designed and fabricated on a silicon photonic platform.

\section{REFERENCES}

[1] A. Samani et al., "A Low-Voltage 35-GHz Silicon Photonic ModulatorEnabled 112-Gb/s Transmission System," IEEE Photonics Journal, vol. 7, no. 3, pp. 1-13, 2015.

[2] D. Vermeulen et al., "Demonstration of Silicon Photonics Push Pull Modulators Designed for Manufacturability," Photonics Technology Letters, vol. 28, no. 10, pp. 1127-1129, 2016.

[3] W. Zhang and J. Yao, "Silicon-Based Integrated Microwave Photonics," in IEEE Journal of Quantum Electronics, vol. 52, no. 1, pp. 1-12, Jan. 2016.

[4] R. Krahenbuhl and M. M. Howerton, "Investigations on short-path-length high-speed optical modulators in $\mathrm{LiNbO} 3$ with resonant-type electrodes," in Journal of Lightwave Technology, vol. 19, no. 9, pp. 1287-1297, 2001. 